\title{
Effects of Strontium Ion Doping on the Thermoelectric Properties of Dysprosium Cobalt Oxide
}

\author{
Hideki Hashimoto $^{1,2, *}$, Takafumi Kusunose ${ }^{1}$ and Tohru Sekino ${ }^{2}$ \\ ${ }^{1}$ The Institute of Scientific and Industrial Research, Osaka University, Ibraki 567-0047, Japan \\ ${ }^{2}$ Institute of Multidisciplinary Research for Advanced Materials, Tohoku University, Sendai 980-8577, Japan
}

\begin{abstract}
We prepared $\mathrm{Dy}_{1-x} \mathrm{Sr}_{x} \mathrm{CoO}_{3-\delta}(x=0,0.025)$ ceramics and evaluated their thermoelectric performances at temperatures up to $873 \mathrm{~K}$. The sample in which $x=0.025$ showed a higher electrical conductivity and a lower Seebeck coefficient than that in which $x=0$. Due to phonon scattering, the thermal conductivity of the $x=0.025$ sample was lower than that of the $x=0$ sample over the entire range measured although the carrier component of thermal conductivity of the $x=0.025$ sample was slightly larger than that of the $x=0$ sample. Due to the effects of an increase of the electrical conductivity and a decrease of the thermal conductivity, the sample in which $x=0.025$ showed the highest figure of merit, $Z=5.7 \times 10^{-5} \mathrm{~K}^{-1}$, at $773 \mathrm{~K}$ in the present system. These results imply that the partial substitution of a larger and lighter divalent cation for the trivalent cation of the A-site effectively enhanced the thermoelectric performance of the $\mathrm{RCoO}_{3}$ system.

[doi:10.2320/matertrans.M2009335]
\end{abstract}

(Received October 1, 2009; Accepted November 10, 2009; Published December 23, 2009)

Keywords: perovskite, dysprosium cobalt oxide, strontium ion, thermoelectric properties

\section{Introduction}

The energy conversion performance of thermoelectric materials is usually characterized by the figure of merit $Z$ or by the dimensionless figure of merit, $Z T$ ( $T$ : absolute temperature).

$$
Z=S^{2} \sigma \kappa^{-1}
$$

where $S$ is the Seebeck coefficient, $\sigma$ is the electrical conductivity, and $\kappa$ is the thermal conductivity. ${ }^{1)}$ There are trade-off relations among these three parameters $(S, \sigma$, and $\kappa)$, so it is difficult to enhance the $Z$ value. In general, a value of $Z T$ greater than 1 is required for the practical application of thermoelectric materials. ${ }^{2,3)}$ Only a few nonoxide materials, such as $\mathrm{Bi}_{2} \mathrm{Te}_{3}$ and $\mathrm{Zn}_{4} \mathrm{Sb}_{3}$, fulfill that requirement; ${ }^{4}$ they have been used in devices such as Peltier coolers. However, they contain toxic elements such as tellurium or antimony and non-oxide materials unstable at high temperatures or under oxidizing conditions. Therefore, oxide compounds that do not contain toxic elements are desirable for use at higher temperatures as thermoelectric materials and in devices used to convert geothermal or waste heat into electrical energy.

Researchers had previously thought that oxide materials did not function in such applications because of their low carrier mobility, i.e., their low electrical conductivity. ${ }^{5)}$ However, since the high thermoelectric performance of $\mathrm{Na}_{x} \mathrm{CoO}_{2}$ was discovered, ${ }^{6}$ ) thermoelectric oxide materials have received much attention. Single crystal $\mathrm{Na}_{x} \mathrm{CoO}_{2}$ exhibits a $Z T$ value higher than $1 .^{7)}$ Although $\mathrm{Na}_{x} \mathrm{CoO}_{2}$ has good thermoelectric performance as an oxide material, it has low chemical and thermal stability, resulting from hydration reactions under humid conditions and decomposition by volatilization of sodium at high temperatures. Thus, stable oxide thermoelectric materials need to be developed.

*Graduate Student, Osaka University and Research Student, Tohoku University
Perovskite-type rare earth cobalt oxides $\left(\mathrm{RCoO}_{3}\right)$ are stable at high temperatures and under oxidizing conditions and do not contain toxic elements. Researchers have reported that among ceramic oxide materials, $\mathrm{RCoO}_{3}$-based ceramics are p-type semiconductors and are promising candidates for thermoelectric oxide materials. ${ }^{8-12)}$

By substituting (doping) a portion of the rare earth ions (trivalent cations) of $\mathrm{RCoO}_{3}$ with divalent cations, we expected that the electrical conductivity would increase. On the other hand, the thermal conductivity $\kappa$ consists of the carrier component $\kappa_{\mathrm{el}}$ and the phonon component $\kappa_{\mathrm{ph}}$ $\left(\kappa=\kappa_{\mathrm{el}}+\kappa_{\mathrm{ph}}\right) ; \kappa_{\mathrm{el}}$ is a function of the electrical conductivity as expressed by the Wiedemann-Franz law,

$$
\kappa_{\mathrm{el}}=L \sigma T
$$

where the Lorenz number $(L)=2.45 \times 10^{-8} \mathrm{WS}^{-1} \mathrm{~K}^{-2}{ }^{8}$ ) Therefore, the increase of the electrical conductivity causes the increase of the thermal conductivity $(\kappa)$ by increasing the carrier component of the thermal conductivity $\left(\kappa_{\mathrm{el}}\right)$ as reported on a Ca-doped $\mathrm{GdCoO}_{3}$ system. ${ }^{10)}$ Meanwhile, the effect of phonon scattering would be expected due to the difference in the ionic radii or the atomic masses between an A-site ion and a substituted ion, and it would cause a decrease of the phonon component of the thermal conductivity $\left(\kappa_{\mathrm{ph}}\right) .{ }^{13,14)}$ Therefore, the thermal conductivity might be controlled by the substitution of A-site ions for ions that have different ionic radii and atomic masses from the original ions. In this study, we focused on the effects of substituting the ions mentioned above and selected $\mathrm{Dy}^{3+}$ [ionic radius, $124 \mathrm{pm} ;{ }^{15)}$ atomic mass, $162.5^{16)}$ ] as the A-site cation of $\mathrm{RCoO}_{3}$ and $\mathrm{Sr}^{2+}\left[144 \mathrm{pm} ;{ }^{15)} 87.62^{16)}\right]$ as the substituent cation. In general, when a solid solution is designed, an ion with a radius close to that of the original ion is selected as the substituent. Although the difference of the ionic radii between $\mathrm{Dy}^{3+}$ and $\mathrm{Sr}^{2+}$ is not small, both ions are constituents of perovskite-type cobalt oxides, $\mathrm{DyCoO}_{3}$ and $\mathrm{SrCoO}_{3-\delta},{ }^{17,18)}$ respectively. Therefore, we believed that the solid solution $\mathrm{Dy}_{1-x} \mathrm{Sr}_{x} \mathrm{CoO}_{3-\delta}$ with better properties 
could be synthesized and selected $\mathrm{Dy}^{3+}$ and $\mathrm{Sr}^{2+}$ ions as for A-site cations, expecting the effects of phonon scattering. The compounds were then synthesized by reaction sintering of the corresponding metal oxide powders, and the effects of substitution of the trivalent $\mathrm{Dy}^{3+}$ ion by the larger and lighter, divalent $\mathrm{Sr}^{2+}$ ion on the electric, thermal, and thermoelectric properties of these materials were investigated at temperatures up to $873 \mathrm{~K}$.

\section{Experimental Procedures}

$\mathrm{Dy}_{2} \mathrm{O}_{3}, \mathrm{SrCO}_{3}$, and $\mathrm{Co}_{3} \mathrm{O}_{4}$ (all $99.9 \%$ purity; Kojundo Chemical Laboratory, Saitama, Japan) were selected as raw materials and weighed in $[(1-x) \mathrm{Dy}+x \mathrm{Sr}] / \mathrm{Co}=1 \quad(x=$ $0,0.025)$ by the atomic ratio. We mixed them by wet and dry ball milling and then calcined the mixed powders at $973 \mathrm{~K}$ for $5 \mathrm{~h}$. The calcined powders were compacted by uniaxial pressing (30 MPa) and cold isostatic pressing (200 MPa) to form disks $15 \mathrm{~mm}$ in diameter and $3 \mathrm{~mm}$ in thickness. We then sintered the disks in air at $1173-1323 \mathrm{~K}(x=0)$ and $1273 \mathrm{~K}(x=0.025)$ for $8 \mathrm{~h}$.

We identified the crystalline phases of the sintered samples by X-ray diffraction method (XRD, RINT 2200; Rigaku, Kyoto, Japan) using the $\mathrm{Cu} \mathrm{K} \alpha$ line. Electrical conductivities were measured using the van der Pauw method (ResiTest 8308; TOYO, Tokyo, Japan) in air at temperatures up to $873 \mathrm{~K}$. We measured the Seebeck coefficients of the samples in air up to $873 \mathrm{~K}$ by the conventional steady state method using the same instrument we used for the electrical conductivity measurements. Thermal diffusivities were measured using the laser flash method (TC-7000; ULVACRIKO, Tokyo, Japan), and the specific heats of the samples were measured by differential scanning calorimetry (DSC) (DSC 404C; NETZSCH, Selb, Germany). We performed both measurements under a high purity Ar atmosphere up to $873 \mathrm{~K}$. The densities of the sintered materials were measured via Archimedes' immersion method using toluene. The thermal conductivities of the samples were then calculated from the products of the thermal diffusivity, specific heat, and density values.

\section{Results and Discussion}

To determine the conditions with which we could obtain single phase perovskite-type $\mathrm{DyCoO}_{3}$ by reaction sintering of the $\mathrm{Co}_{3} \mathrm{O}_{4}$ and $\mathrm{Dy}_{2} \mathrm{O}_{3}$ powders, the compacted powders with $x=0$ were sintered at $1173-1323 \mathrm{~K}$ with $50 \mathrm{~K}$ intervals. By XRD measurements, we identified the samples sintered at 1173,1223 , and $1273 \mathrm{~K}$ as orthorhombic perovskite-type DyCoO 3 ; the results were in good agreement with values in the JCPDS-International Centre for Diffraction Data. ${ }^{19)}$ On the other hand, we identified traces of $\mathrm{Dy}_{2} \mathrm{O}_{3}$ in $\mathrm{DyCoO}_{3}$, in the sample sintered at $1323 \mathrm{~K}$, indicating that $\mathrm{DyCoO}_{3}$ decomposed above $1323 \mathrm{~K}$. Therefore, we selected $1273 \mathrm{~K}$ as the sintering temperature of the preparation of the sample with $x=0.025$. The sample with $x=0.025$ was single phase and had a perovskite-type structure similar to $\mathrm{DyCoO}_{3}$, and the peaks of the XRD pattern of the sample with $x=0.025$ slightly shifted to the low angle side compared with that of the $x=0$ sample. By calculating the lattice constants and the

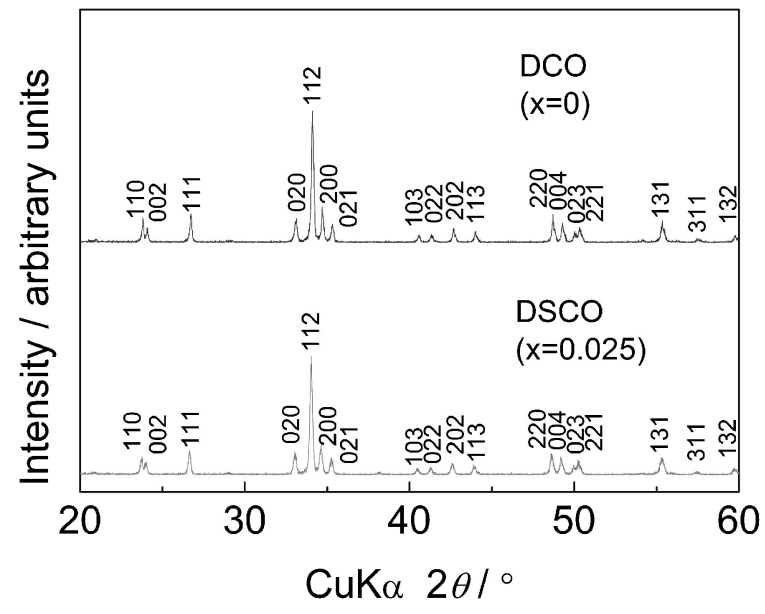

Fig. 1 X-ray diffraction patterns of DCO and DSCO.

Table 1 Lattice constants and unit cell volumes of DCO and DSCO.

\begin{tabular}{ccccc}
\hline & $a / \mathrm{nm}$ & $b / \mathrm{nm}$ & $c / \mathrm{nm}$ & $V / \mathrm{nm}^{3}$ \\
\hline DCO & 0.5166 & 0.5408 & 0.7389 & 0.2064 \\
DSCO & 0.5168 & 0.5411 & 0.7411 & 0.2072 \\
\hline
\end{tabular}

unit cell volumes using the XRD data, we confirmed that the unit cell volume of the $x=0.025$ sample was larger than that of the $x=0$ sample. The increase of the unit cell volume might be caused by increasing the average ionic radii of the A-site of $\mathrm{DyCoO}_{3}$ with doped $\mathrm{Sr}^{2+}$ ions. [The unit cell volume of $\mathrm{RCoO}_{3}$ increased with the increasing ionic radii of the A-site ions. ${ }^{19,20)}$ ] Therefore, we believed that the $\mathrm{Sr}^{2+}$ ions were doped to the A-sites of $\mathrm{DyCoO}_{3}$. As to the sintered density (the relative density) of each sample sintered at $1273 \mathrm{~K}$, Sr-doping tended to cause a decrease of the density. The sample with $x=0$ was $5.57 \mathrm{gcm}^{-3}(63 \%)$, and that with $x=0.025$ was $4.10 \mathrm{gcm}^{-3}(47 \%)$. On the other hand, the density (the relative density) of the $x=0$ sample sintered at $1173 \mathrm{~K}$ was $4.27 \mathrm{gcm}^{-3}$ (49\%). Therefore, we selected those two samples, prepared as $x=0$ sintered at $1173 \mathrm{~K}$ and as $x=0.025$ sintered at $1273 \mathrm{~K}$, to compare their properties due to their similar relative densities. In the following, each sample is abbreviated as DCO and DSCO, respectively. Figure 1 and Table 1 show the XRD patterns of DCO and DSCO, and the lattice constant and the unit cell volume of DCO and DSCO, respectively.

Figure 2(a) shows the Arrhenius plot of the electrical conductivities of DCO and DSCO. The electrical conductivities of both samples showed semiconductive conduction. The electrical conductivity of DSCO was higher than that of DCO due to the carrier doping effect. Although it was effective over the entire range measured, the effect tended to decrease with increasing temperature. Figure 2(b) shows the temperature dependence of the quantities $-k_{\mathrm{B}} \Delta \ln \sigma / \Delta\left(T^{-1}\right)$ of DCO and DSCO calculated from the electrical conductivity data (the Arrhenius plot of Fig. 2(a)) using the forward difference formula in which $k_{\mathrm{B}}$ is the Boltzmann constant. When the mechanism is simple thermal activation-type conduction, the value corresponds to the activation energy, and it does not depend on the temperature. On the other hand, 
(a)

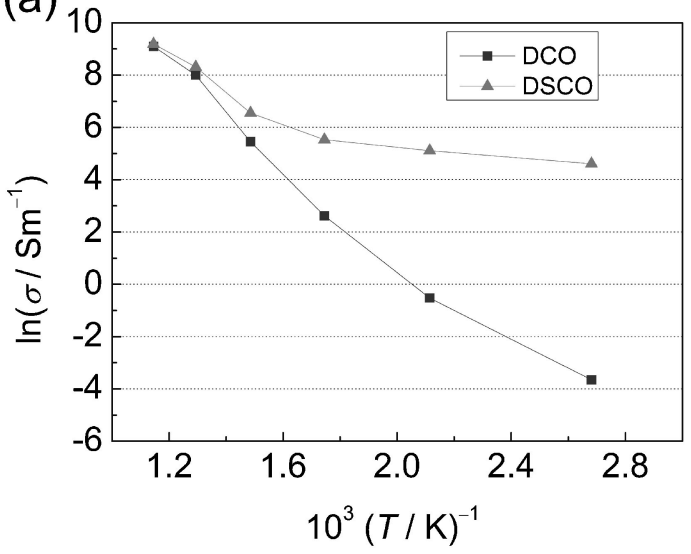

(b)

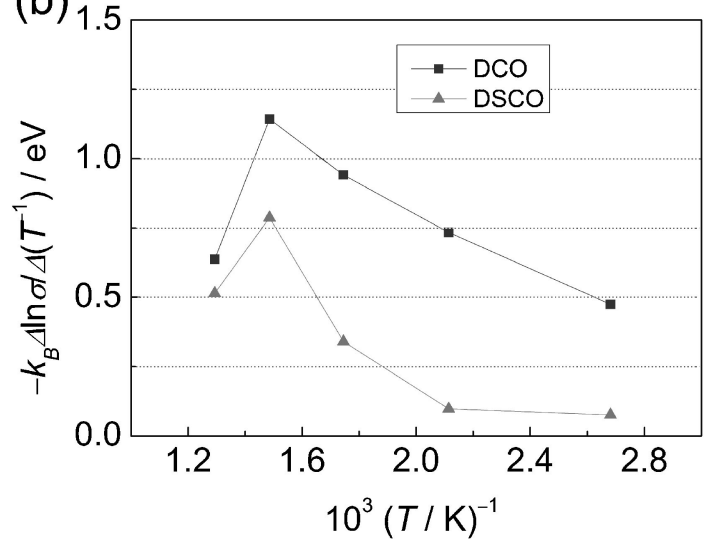

Fig. 2 Arrhenius plot of electrical conductivities of DCO and DSCO (a), and the quantities $\left(-k_{\mathrm{B}} \Delta \ln \sigma / \Delta\left(T^{-1}\right)\right)$ of DCO and DSCO (b) calculated from the Arrhenius plot of the electrical conductivities.

in the present system, the values depended on the temperature; they increased with increasing temperature up to a maximum and then decreased. Therefore, we assumed that several conduction mechanisms coexisted within the present test temperature region. Another important characteristic illustrated by this figure is that the $-k_{\mathrm{B}} \Delta \ln \sigma / \Delta\left(T^{-1}\right)$ curves show maxima. For the $\mathrm{RCoO}_{3}$ system, researchers have reported that the maximum indicates the steep metalinsulator crossover. ${ }^{21}$ ) Therefore, DCO (and DSCO) tended to transit from insulator to metal with increasing temperature, and the effects of $\mathrm{Sr}^{2+}$ ion doping on the electrical conductivities decreased with increasing temperature.

Figure 3 shows the Seebeck coefficients $(S)$ of DCO and DSCO. Since the values of $S$ for the entire system were positive over the entire range measured, the major conduction carriers were holes, thus indicating p-type semiconductors. These results corresponded to the fact that $\mathrm{RCoO}_{3}$ are p-type semiconductors as described in previous reports. ${ }^{8-12)}$ The Seebeck coefficients of these samples decreased with increasing temperature, and at a constant temperature they increased with increasing $x$ values. Generally, in semiconductors, the Seebeck coefficient tends to decrease when the electrical conductivity increases with increasing carrier concentration; this trend was also true for the present case with the trade-off relationship between the electrical conductivity and the Seebeck coefficient.

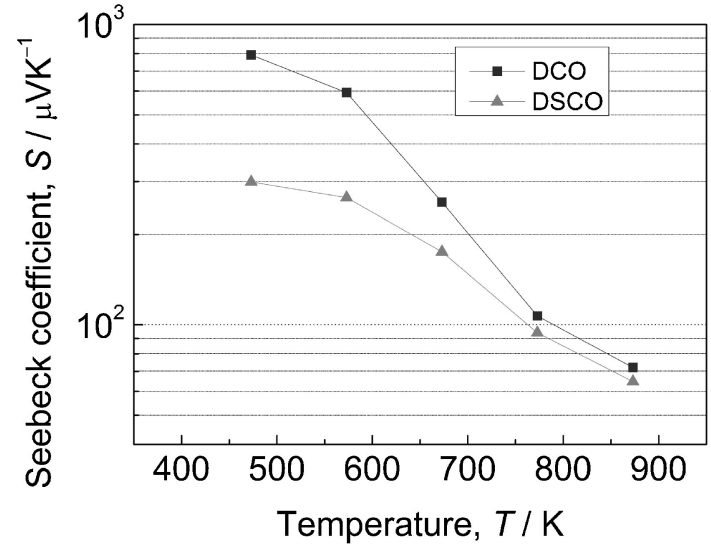

Fig. 3 Temperature dependence of the Seebeck coefficients of DCO and DSCO.

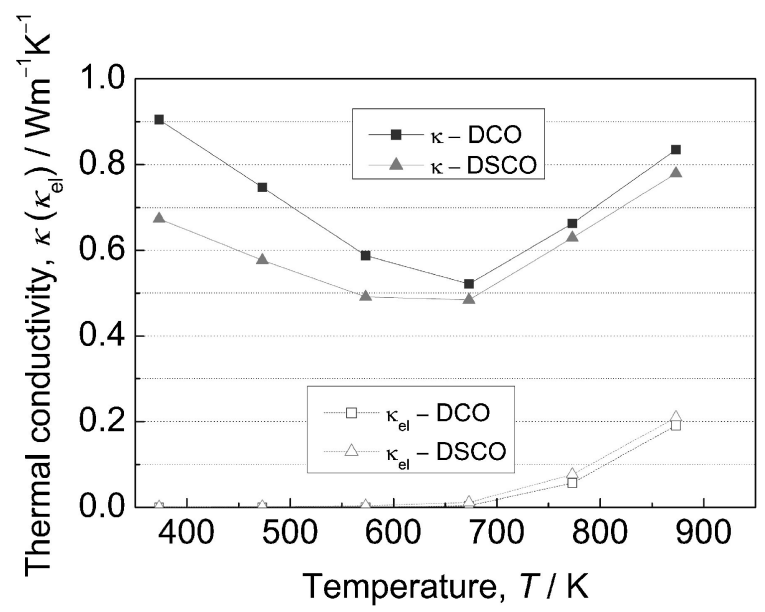

Fig. 4 Temperature dependence of the measured thermal conductivities $(\kappa)$ and electronic contributions of the thermal conductivities $\left(\kappa_{\mathrm{el}}\right)$ calculated from the electrical conductivity data by the Wiedemann-Franz law for DCO and DSCO.

Figure 4 shows the thermal conductivities $(\kappa)$ and the carrier components of the thermal conductivities $\left(\kappa_{\mathrm{el}}\right)$ of DCO and DSCO. On the low temperature side, the thermal conductivities of both samples decreased with increasing temperature; this trend was caused by the increase of phonon scattering with increasing temperature. The thermal conductivity of DSCO was lower than that of DCO although the difference gradually decreased with increasing temperature; this trend was caused by the effect of increasing phonon scattering by the substitution of $\mathrm{Sr}^{2+}$ for $\mathrm{Dy}^{3+}$. On the high temperature side, the thermal conductivities of both samples increased with increasing temperature, corresponding to the increase of the electrical component of the thermal conductivity $\left(\kappa_{\mathrm{el}}\right)$ with increasing temperature. $\kappa_{\mathrm{el}}$ of DSCO was slightly larger than that of DCO whereas the opposite was confirmed for $\kappa$. The tendency of $\kappa$ was due to the lower $\kappa_{\mathrm{ph}}$ of DSCO mentioned above. As a result, the thermal conductivity $(\kappa)$ of DSCO was lower than that of DCO over the entire range measured.

Figure 5 shows the calculated figures of merit $(Z)$. At $873 \mathrm{~K}$, the $Z$ of DSCO was smaller than that of DCO. On the other hand, up to $773 \mathrm{~K}$, the $Z$ of DSCO was larger than that 


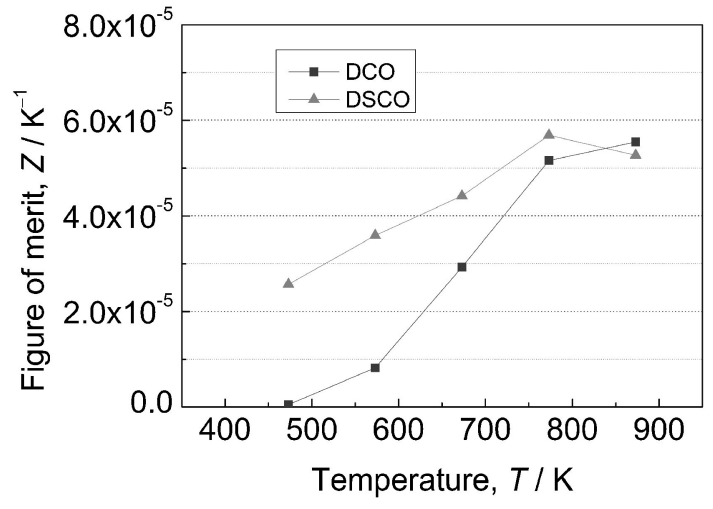

Fig. 5 Temperature dependence of the figures of merit of DCO and DSCO.

of DCO due to the effects of an increase of the electrical conductivity and a decrease of the thermal conductivity. In the present system, the sample of DSCO showed the largest figure of merit, $Z=5.7 \times 10^{-5} \mathrm{~K}^{-1}$ at $773 \mathrm{~K}$.

\section{Conclusion}

We prepared perovskite-type rare earth cobalt oxide $\mathrm{Dy}_{1-x} \mathrm{Sr}_{x} \mathrm{CoO}_{3-\delta} \quad(x=0$ and 0.025 ; abbreviated to DCO and DSCO, respectively) by reaction sintering of the corresponding metal oxide powders and evaluated their thermoelectric performance at temperatures up to $873 \mathrm{~K}$. By the partial substitution of divalent $\mathrm{Sr}^{2+}$ ions for trivalent $\mathrm{Dy}^{3+}$ ion, the electrical conductivity of DSCO was greater than that of DCO. The effect tended to decrease with increasing temperature; it was related to the metal-insulator transition due to temperature dependence. The Seebeck coefficients showed the opposite tendency to that of the electrical conductivities. The thermal conductivity of DSCO was less than that of DCO over the entire range measured due to the effects of phonon scattering by substitution of the larger and lighter $\mathrm{Sr}^{2+}$ for $\mathrm{Dy}^{3+}$, although it had the larger thermal conductivity of the carrier component $\left(\kappa_{\mathrm{el}}\right)$. These results indicated that the increase of $\kappa_{\mathrm{el}}$ could be compensated for by the decrease of $\kappa_{\mathrm{ph}}$ due to phonon scattering by selecting a suitable substituent cation and that this compensation was effective in decreasing the thermal conductivity of DCO by substituting $\mathrm{Sr}^{2+}$ for $\mathrm{Dy}^{3+}$, especially on the low temperature side. Due to the effects of the increase of the electrical conductivity and the decrease of the thermal conductivity, DSCO showed the highest figure of merit, $Z=5.7 \times 10^{-5} \mathrm{~K}^{-1}$ at $773 \mathrm{~K}$ in the present system. These results implied that the partial substitution of a larger and lighter divalent cation (such as $\mathrm{Sr}^{2+}$ ) for the trivalent cation (such as $\mathrm{Dy}^{3+}$ ) of the A-sites effectively enhanced the thermoelectric performance of the $\mathrm{RCoO}_{3}$ system.

\section{REFERENCES}

1) G. Chen, M. S. Dresselhaus, G. Dresselhaus, J.-P. Fleurial and T. Caillat: Int. Mater. Rev. 48 (2003) 45-66.

2) S. Ohta, T. Nomura, H. Ohta and K. Koumoto: J. Appl. Phys. 97 (2005) 034106.

3) K. Kurosaki, T. Sekimoto, K. Kawano, H. Muta and S. Yamanaka: J. Jpn. Powder Powder Metall. 54 (2007) 370-374.

4) T. Caillat, J.-P. Fleurial and A. Borshchevsky: J. Phys. Chem. Solids 58 (1997) 1119-1125.

5) N. Murayama and K. Koumoto: Bull. Ceram. Soc. Jpn. 33 (1998) 161165.

6) I. Terasaki, Y. Sasago and K. Uchinokura: Phys. Rev. B 56 (1997) R12685-R12687.

7) K. Fujita, T. Mochida and K. Nakamura: Jpn. J. Appl. Phys. 40 (2001) 4644-4647.

8) J.-W. Moon, W.-S. Seo, H. Okabe, T. Okawa and K. Koumoto: J. Mater. Chem. 10 (2000) 2007-2009.

9) J.-W. Moon, Y. Masuda, W.-S. Seo and K. Koumoto: Mater. Lett. 48 (2001) 225-229.

10) J.-W. Moon, Y. Masuda, W.-S. Seo and K. Koumoto: Mater. Sci. Eng. B 85 (2001) 70-75.

11) H. Hashimoto, T. Kusunose and T. Sekino: J. Alloy. Compd. 484 (2009) 246-248.

12) W. Wunderlich and H. Fujiwara: Proc. Int. Conf. Thermoelectric (2009) arxiv: 0909.1618.

13) W. D. Kingery, H. K. Bowen and D. R. Uhlmann: Introduction to Ceramics, 2nd edition, (Wiley Interscience, New York, 1976) pp. 615624.

14) N. P. Padture and P. G. Klemens: J. Am. Ceram. Soc. 80 (1997) 10181020.

15) Y. Q. Jia: J. Solid State Chem. 95 (1991) 184-187.

16) International Union of Pure and Applied Chemistry: Quantities, Units, and Symbols in Physical Chemistry, 3rd edition, (RSC Publishing, Cambridge, 2007) pp. 121-128.

17) JCPDS-International Centre for Diffraction Data, PDF No. 39-1083, 39-1084, and 40-1018.

18) S. Balamurugan, K. Yamaura, A. B. Karki, D. P. Young, M. Arai and E. Takayama-Muromachi: Phys. Rev. B 74 (2006) 172406.

19) JCPDS-International Centre for Diffraction Data, PDF No. 25-1051.

20) JCPDS-International Centre for Diffraction Data, PDF No. 25-1069, 25-1064, 25-1071, 25-1054, 25-1057, and 25-1073.

21) S. Yamaguchi, Y. Okimoto and Y. Tokura: Phys. Rev. B 54 (1996) R11022. 